\title{
Pitfalls in PSMA-PET/CT: intensive bone marrow uptake in a case with polycythemia vera
}

\author{
Philipp E. Hartrampf ${ }^{1}$ (D) $\cdot$ Bernhard Petritsch ${ }^{2} \cdot$ Andreas K. Buck $^{1} \cdot$ Sebastian E. Serfling ${ }^{1}$
}

Received: 7 September 2020 / Accepted: 12 October 2020 / Published online: 28 October 2020

(C) The Author(s) 2020

A patient with Gleason 6 prostate carcinoma (initial diagnosis April 2012) underwent $\left[{ }^{18} \mathrm{~F}\right] \mathrm{PSMA} 1007 \mathrm{PET} / \mathrm{CT}$ for restaging. After several years of watch and wait, bone metastases were detected in November 2017 with bone scintigraphy (oligometastatic disease), and anti-androgenic therapy was initiated. PSA values initially dropped, then after significant PSA relapse in March 2019 (PSA value $153 \mathrm{ng} / \mathrm{ml}$ ), a secondline antihormonal therapy with abiraterone was started. Restaging with bone scintigraphy and MRI in June 2020 revealed contradictory findings. Whereas MRI findings suggested multifocal disease, the bone scan still indicated oligometastatic bone disease. For clarification, $\left[{ }^{18} \mathrm{~F}\right] \mathrm{PSMA}$ $1007 \mathrm{PET} / \mathrm{CT}$ was performed. PSA value at the time of imaging was $0.2 \mathrm{ng} / \mathrm{ml}$. Additionally, the patient has been suffering from polycythemia vera (PV) for about 20 years, which is currently under therapy with hydroxyurea since December 2019. Splenectomy has been performed already in September 2019. $\left[{ }^{18} \mathrm{~F}\right]$ PSMA 1007 PET/CT showed intense tracer uptake in the bone marrow compartment of the entire skeleton with few focal spots (A). A small accessory spleen also showed an intensive tracer uptake (A, black arrow). T1weighted MRI of the vertebral column revealed a signal loss in vertebral bodies to the level of spinal discs, indicating a

This article is part of the Topical Collection on Image of the month

Philipp E. Hartrampf

Hartrampf_P@ukw.de

1 Department of Nuclear Medicine, University Hospital Würzburg, Würzburg, Germany

2 Department of Diagnostic and Interventional Radiology, University Hospital Würzburg, Würzburg, Germany replacement of fatty bone marrow by the red bone marrow (B). Metastatic thoracic vertebra 11 presents without tracer uptake (B, C, blue arrows). Furthermore, CT revealed multiple osteoblastic lesions in the entire skeleton but without relevant tracer uptake (D, E, red arrows), indicating disease control or non-viable bone metastases under current antihormonal therapy with abiraterone, as shown recently by Plouznikoff et al. for patients receiving novel antiandrogen drugs [1]. In conclusion, increased $\left[{ }^{18}\right.$ F]PSMA 1007 uptake in the bone marrow compartment is most likely caused by PV, rather than bone metastases from prostate cancer.

Literature research revealed a sole case report with only mild diffuse bone marrow $\left[{ }^{18} \mathrm{~F}\right] \mathrm{PSMA}$ uptake in a patient with polycythemia rubra vera [2]. In our patient, $\left[{ }^{18} \mathrm{~F}\right]$ PSMA 1007 uptake in the bone marrow compartment could be caused by the onset of myelofibrosis (MF), as the patient has been suffering from PV for several years before. Increased vascularity is assumed in PV and especially in MF [3]. Lundberg et al. have shown that myeloproliferative diseases, such as PV and MF in particular, show activation of neoangiogenesis [4]. Since PSMA is also expressed in the neovasculature of some tumors other than prostate cancer [5], binding to the neovasculature could occur in PV or MF, leading to nonspecific tracer uptake in the bone marrow.

Our case demonstrates that $\left[{ }^{18} \mathrm{~F}\right] \mathrm{PSMA} 1007$ uptake in the bone marrow can be very intense in PV, a finding that may be misinterpreted as diffuse bone metastases from prostate cancer. Although PV is a rare disease, detailed medical history is important for differential diagnosis of intense $\left[{ }^{18} \mathrm{~F}\right] \mathrm{PSMA}$ 1007 uptake in the bone marrow. Especially at very low PSA values or PSA values inadequate for extensive disease, one should consider the presence of hematological diseases as differential diagnosis. 

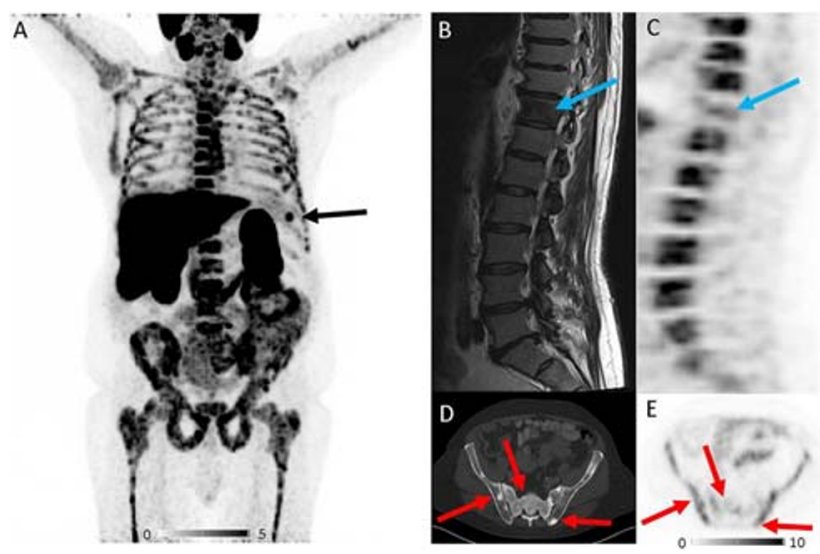

Funding Open Access funding enabled and organized by Projekt DEAL.

\section{Compliance with ethical standards}

This is a case report from clinical practice. No trial registration or approval from the institutional research committee is needed for this kind of publication.

Conflict of interest The authors declare that they have no conflict of interest.

Informed consent Informed consent was obtained from the patient included in the study. Patient identity in this retrospective single case report has been fully anonymized to comply with privacy standards.

Open Access This article is licensed under a Creative Commons Attribution 4.0 International License, which permits use, sharing, adaptation, distribution and reproduction in any medium or format, as long as you give appropriate credit to the original author(s) and the source, provide a link to the Creative Commons licence, and indicate if changes were made. The images or other third party material in this article are included in the article's Creative Commons licence, unless indicated otherwise in a credit line to the material. If material is not included in the article's Creative Commons licence and your intended use is not permitted by statutory regulation or exceeds the permitted use, you will need to obtain permission directly from the copyright holder. To view a copy of this licence, visit http://creativecommons.org/licenses/by/4.0/.

\section{References}

1. Plouznikoff N, Artigas C, Sideris S, Martinez Chanza N, Gil T, Peltier A, et al. Evaluation of PSMA expression changes on PET/ CT before and after initiation of novel antiandrogen drugs (enzalutamide or abiraterone) in metastatic castration-resistant prostate cancer patients. Ann Nucl Med. 2019;33:945-54. https://doi.org/ 10.1007/s12149-019-01404-2.

2. Shetty D, Patel D, Le K, Bui C, Mansberg R. Pitfalls in gallium-68 PSMA PET/CT interpretation-a pictorial review. Tomography. 2018;4:182-93. https://doi.org/10.18383/j.tom.2018.00021.

3. Thiele J, Rompcik V, Wagner S, Fischer R. Vascular architecture and collagen type IV in primary myelofibrosis and polycythaemia vera: an immunomorphometric study on trephine biopsies of the bone marrow. Br J Haematol. 1992;80:227-34. https://doi.org/10. 1111/j.1365-2141.1992.tb08905.x.

4. Lundberg LG, Lerner R, Sundelin P, Rogers R, Folkman J, Palmblad $\mathrm{J}$. Bone marrow in polycythemia vera, chronic myelocytic leukemia, and myelofibrosis has an increased vascularity. Am J Pathol. 2000;157:15-9. https://doi.org/10.1016/s0002-9440(10)64511-7.

5. O'Keefe DS, Bacich DJ, Huang SS, Heston WDW. A perspective on the evolving story of PSMA biology, PSMA-based imaging, and endoradiotherapeutic strategies. J Nucl Med. 2018;59:1007-13. https://doi.org/10.2967/jnumed.117.203877.

Publisher's note Springer Nature remains neutral with regard to jurisdictional claims in published maps and institutional affiliations. 